\title{
CANTAB object recognition and language tests to detect aging cognitive decline: an exploratory comparative study
}

\author{
Fernanda Cabral Soares' \\ Thaís Cristina Galdino de \\ Oliveira' \\ Liliane Dias e Dias de \\ Macedo' \\ Alessandra Mendonça \\ Tomás' \\ Domingos Luiz Wanderley \\ Picanço-Diniz ${ }^{2}$ \\ João Bento-Torres ${ }^{1,3}$ \\ Natáli Valim Oliver Bento- \\ Torres ${ }^{1,3}$ \\ Cristovam Wanderley \\ Picanço-Diniz' \\ 'Universidade Federal do Pará, \\ Instituto de Ciências Biológicas, \\ Hospital Universitário João de Barros \\ Barreto, Laboratório de Investigações \\ em Neurodegeneração e Infecção \\ Belém, Pará, Brazil; ${ }^{2}$ Universidade \\ Federal do Oeste do Pará, Núcleo \\ Universitário de Oriximiná, Oriximiná, \\ Pará, Brazil; ${ }^{3}$ Faculdade de Fisioterapia \\ e Terapia Ocupacional, Universidade \\ Federal do Pará, Belém, Pará, Brazil
}

Correspondence: Cristovam Wanderley Picanço-Diniz

Hospital Universitário João de Barros Barreto, Laboratório de Investigações em Neurodegeneração e Infecção, Rua Dos Mundurucus 4487, 66073005, Belém, Pará, Brazil

Tel +55 9| 320। 6756

Fax +55 9l 320I 6756

Email cwpdiniz@gmail.com
This article was published in the following Dove Press journal:

Clinical Interventions in Aging

19 December 2014

Number of times this article has been viewed

\begin{abstract}
Objective: The recognition of the limits between normal and pathological aging is essential to start preventive actions. The aim of this paper is to compare the Cambridge Neuropsychological Test Automated Battery (CANTAB) and language tests to distinguish subtle differences in cognitive performances in two different age groups, namely young adults and elderly cognitively normal subjects.
\end{abstract}

Method: We selected 29 young adults (29.9 \pm 1.06 years) and 31 older adults $(74.1 \pm 1.15$ years) matched by educational level (years of schooling). All subjects underwent a general assessment and a battery of neuropsychological tests, including the Mini Mental State Examination, visuospatial learning, and memory tasks from CANTAB and language tests. Cluster and discriminant analysis were applied to all neuropsychological test results to distinguish possible subgroups inside each age group.

Results: Significant differences in the performance of aged and young adults were detected in both language and visuospatial memory tests. Intragroup cluster and discriminant analysis revealed that $\mathrm{CANTAB}$, as compared to language tests, was able to detect subtle but significant differences between the subjects.

Conclusion: Based on these findings, we concluded that, as compared to language tests, largescale application of automated visuospatial tests to assess learning and memory might increase our ability to discern the limits between normal and pathological aging.

Keywords: aging, neuropsychological tests, cluster analysis, discriminant analysis

\section{Introduction}

The increasing rate of chronic neurodegenerative diseases in the elderly, such as Alzheimer's disease (AD), is a global phenomenon. Because AD occurs in both developed and developing countries and contributes to $70 \%$ of all reported dementias, ${ }^{1}$ it has become a driving force in aging research. To reduce its impact on the public health budget, ${ }^{2,3}$ early diagnosis and prevention of cognitive decline may be essential. ${ }^{4}$ In this context, the distinction between individuals who will maintain adequate cognitive function into their later years and those on a trajectory to dementia is a fundamental question being pursued..$^{5}$ It is essential to establish accurate and sensitive tools for assessing the cognitive function and the neuropsychological screening of the aging population.

The Mini Mental State Examination (MMSE) ${ }^{6}$ is the most widely used cognitive screening tool in both clinical settings and epidemiological studies. ${ }^{7}$ However, the MMSE is unable to detect subtle declines in cognitive aging. ${ }^{8}$ It exhibits a ceiling effect in healthy young or older adults and a floor effect in the oldest older adults..$^{9,10}$ Additionally, correcting the MMSE score for educational level influences these 
effects. $^{7}$ In contrast, evidence indicates that performances on tests of episodic memory are significantly lower among mildly impaired but non-demented individuals, who will subsequently fulfill criteria for AD over time. ${ }^{1-17}$ In addition, it has been reported that lower performances on language memory tests may predict cognitive decline. Indeed, semantic memory, ${ }^{17}$ word and story recall, object naming, and Boston Naming Test, ${ }^{18,19}$ as well as semantic, verb, and letter fluencies, ${ }^{20}$ may be predictive for future dementia in aged individuals with mild cognitive impairment. Moreover, automated semantic indices or latent profile assessment based on patterns of cognition from large samples may distinguish preclinical phenotypes for AD. ${ }^{21,22}$ Finally, automated visuospatial learning and memory tests have also shown predictive value regarding future dementia of the Alzheimer type. ${ }^{23-25}$ Compared with traditional standard methods, automated computer tests minimize floor and ceiling effects, standardize the format of application, and measure the speed and accuracy of responses with greater sensitivity. ${ }^{26}$ Among the automated test batteries currently in use, the Cambridge Neuropsychological Test Automated Battery (CANTAB) is frequently applied to discriminate between cognitively healthy aged adults and those in the preclinical stages of future dementia. ${ }^{26,27}$ For example, baseline results from non-demented patients who underwent the paired-associates learning test from the CANTAB were found to correlate with preclinical AD ${ }^{17,23,28,29}$ However, less has been done to compare the resolution of the CANTAB's automated neuropsychological tests with standard neuropsychological tests, in which a few automated scores and normative comparison of raw test scores are available across lifespan, and test/retest studies reported significant variability across administrations. ${ }^{27,30,31}$ CANTAB is a nonverbal visuospatial stimulus battery that employs touchscreen technology to obtain nonverbal responses from participants. It is quite adequate for cognitive assessment across cultures, in both longitudinal and cross-sectional studies, with minimal interference of the researcher or clinician during data acquisition. $^{32}$

Thus, in this study, we have carried out an exploratory investigation to assess the performance of selected CANTAB neuropsychological tests by adult (20-40 years old) and aged ( $\geq 65$ years old) individuals to distinguish intragroup episodic memory performances.

To that end, all individuals were preliminarily submitted to MMSE, and cognitively healthy individuals were selected for language and CANTAB visuospatial memory tests. Cluster and discriminant analyses were applied to the results of each test in isolation or in combination to identify the best choice to distinguish intragroup cognitive performances. Finally, we tested the hypothesis that CANTAB indices would correlate with performances on language tests that assessed the corresponding cognitive domains.

Thus, we address two simple questions: 1) To what extents do language and CANTAB tests, in isolation or in combination, distinguish possible clusters of cognitive performances in young adults and elderly normal subjects? 2) What is the relationship between performances on language tests and CANTAB tests that assess corresponding cognitive domains?

\section{Methods \\ Study participants}

We performed a cross-sectional neuropsychological evaluation of 60 individuals grouped as older aged $(74.1 \pm 1.15$ years, $\mathrm{n}=31)$ and young adults $(29.9 \pm 1.06$ years, $\mathrm{n}=29)$ with no history of head trauma, stroke, primary depression, or chronic alcoholism. All participants had normal cognitive performance when administered the MMSE, with the necessary adjustments for education level according to the criteria for the Brazilian population with the following cutoffs: illiterate, 13 points; $1-7$ years of schooling, 18 points; $\geq 8$ years of schooling, 26 points. ${ }^{33}$ All patients who met these criteria were assessed with selected language, visuospatial learning, and memory tests, as well as the MMSE. The local Research Ethics Committee (protocol No 3955/09) approved this study, and all ethical recommendations in research involving human subjects were observed.

\section{Cognitive evaluation}

We applied the MMSE ${ }^{6,33,34}$ followed by language tests on the same day. The CANTAB test was administered separately on another day. Trained investigators administered these tests in an environment with adequate lighting and reduced noise conditions.

\section{Language assessment}

The Boston Naming Test (shortened version) was administered and analyzed according to parameterized data for Brazil, ${ }^{35,36}$ adopting a cutoff equivalent to the correct naming of 12 of 15 possible figures.

Semantic (SVF) and Phonological Verbal Fluency (PVF) tests were administered and computed using the following cutoffs: $<9$ points for illiterate individuals, $<12$ points for individuals with $1-7$ years of schooling, and $<13$ points for individuals with $\geq 8$ years of schooling. ${ }^{37}$ 
The Test of Narrative "Cookie Theft" was evaluated using previously published criteria on the information content of the image, including the number of key concepts, narrative efficiency, number of units of information, the total number of words, and the concision ratio (ratio of information units to the total number of words). ${ }^{38,39}$

The Montréal d'Evaluation de la Communication is a battery comprised of the following tests: Metaphors (explanation and alternatives), Direct Speech Acts and Indirect Speech Acts (explanation and alternatives), Linguistic and Emotional Prosody, and Narrative Discourse (partial retelling, total retelling, and full text comprehension). The Montréal d'Evaluation de la Communication battery was administered and the performance measured in accordance with guidelines validated for the Brazilian population. $^{40,41}$

\section{Cambridge Neuropsychological Test Automated Battery}

The CANTAB focuses on three cognitive domains: working memory and planning, attention, and visuospatial memory. Responses are via touchscreen and are largely independent of verbal instruction. An XGA-touch panel 12-inch monitor (Paceblade Slim-book P120; PaceBlade Technology, Hong Kong, People's Republic of China) was used to present all visuospatial stimuli for testing. The monitor was controlled using CANTAB software (Cambridge Cognition, Cambridge, UK) run on the slimbook. All study participants were assessed individually. We started with a motor screening task which introduced the CANTAB touchscreen to the individuals. The motor screening task provides a general assay that tests whether sensorimotor or other difficulties limit the collection of valid data from each participant. After touchscreen adaptation, we selected a number of CANTAB tests as follows: Rapid Visual Information Processing (RVP), Reaction Time (RTI), Paired Associate Learning (PAL), Spatial Working Memory (SWM), and Delayed Matching to Sample (DMS). The RVP measure is sensitive to sustained attention. In this test, a white box appears in the center of the screen with digits from 2-9 in a pseudorandom order at the rate of 100 digits per minute, and the subjects are requested to identify target sequences of three digits and to register responses using the press pad. RTI provides assays of motor and mental response speeds, as well as measures of movement time, reaction time, response accuracy, and impulsivity. This test is divided into five stages that are characterized by increasingly complex chains of responses.
PAL assesses visual memory and new learning. In this test, six boxes are displayed on the screen, and they are opened in random order to reveal the contents. One or more of them will contain a pattern. The patterns inside the boxes are then displayed in the middle of the screen one at a time, and the participants must touch the box in which the pattern was originally located. SWM measures the retention and manipulation of visuospatial information. In this test, the participant must search the boxes on the screen to find a blue "token" in each of a number of boxes, and use them to fill an empty column on the right-hand side of the screen; the number of boxes is gradually increased until it is necessary to search eight boxes. DMS assesses forced choice recognition memory for visual patterns, and tests both simultaneous matching and short-term visual memory. In this test, a complex visual pattern (the sample) is shown to the participant, followed by four similar patterns after a short delay. The participant must touch the pattern that exactly matches the sample. For detailed information, see http://www.cambridgecognition.com/clinicaltrials/ cantabsolutions/tests

\section{Results}

We subjected all test results from all participants to an initial cluster analysis (tree clustering method, Euclidean distances, and complete linkage). Cluster analysis encompasses a number of different classification algorithms applied to a wide variety of research problems. ${ }^{42-45}$ They are mostly used when one does not have an a priori hypothesis about which objects belong to a specific cluster group. We applied this multivariate statistical procedure to our sample of study participants in combination (young adults + aged subjects) to search for potential groups of distinct cognitive performances. After that, similar procedures were applied to young adult and aged groups in isolation to search for potential subgroups. The performance groups suggested by cluster analysis were further assessed through the performance of a discriminant function analysis using Statistica 7.0 (Statsoft, Tulsa, OK, USA). Discriminant function analysis is used to determine which variables discriminate between two or more naturally occurring groups. The strategy underlying this procedure is to determine whether groups differ with regard to the mean of a variable, and then to use that variable to predict group membership. In the present study, we performed comparisons between matrices of total variances and covariances. These matrices were compared via multivariate $F$-tests to determine whether there were any significant between-group differences (with regard to all variables). 


\section{Neuropsychologial performances across life span: cluster and discriminant analysis}

Figure $1(\mathrm{~A}-\mathrm{C})$ illustrates four dendrograms resulting from cluster analysis applied to isolated or combined neuropsychological test results of the whole sample, including young and older subjects. Note that, compared with all other possibilities of cluster analysis, either in isolation (Language, Figure 1B) or in combinations of two (CANTAB + Language; Figure $1 \mathrm{C}$ ), the cluster analysis applied to CANTAB results (Figure 1A) discriminated best between the performances of young adults and older adults.

We applied this procedure to determine which neuropsychological tests, isolated or associated, provided the best separation between the performance groups suggested by the cluster analysis. Table 1 shows the results of probability density ( $P$-values) after discriminant analysis. Note that "latency values" on CANTAB RVP was by far the variable that best contributed to cluster formation, in isolation $\left(P=1.0 \times 10^{-10}\right)$ or in combination (CANTAB + Language, $P=4 \times 10^{-12}$ ). Although the cluster formation based on isolated language tests did not discriminate between age groups as well as CANTAB in isolation $\left(P=1 \times 10^{-10}\right)$, metaphors $\left(P=4 \times 10^{-8}\right)$, or emotional prosody $(P=0.021)$, it contributed significantly to cluster formation.

Figure 2 shows graphical representations of discriminant analysis to illustrate the best separation between groups. As compared to isolated CANTAB (Figure 2A) or Language (Figure 2B) isolated tests, discriminant analysis of $\mathrm{CANTAB}$ and language tests results in combination (Figure 2C) provided the best separation between young and older adult's performances. However, the CANTAB results better distinguished young and older adults' performances than language tests. Note that RVP latency, concision ratio, SWM strategy, and information units were the variables that better contributed to cluster formation when combining language and CANTAB test results (Figure 2D). For isolated CANTAB or language tests results, RVP latency and metaphors results, respectively, were by far the variables that best contributed to distinguish young and older adults' performances (Figure 2D).
A

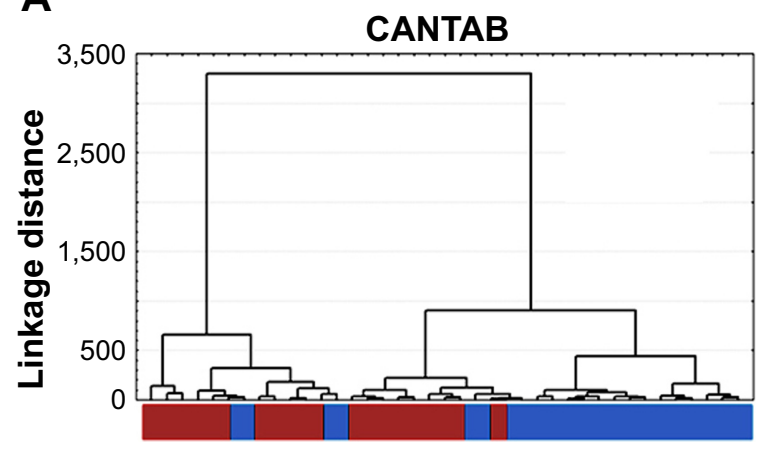

B

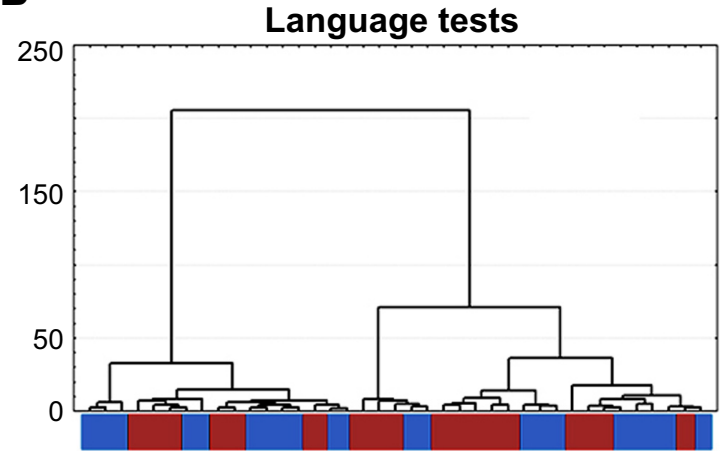

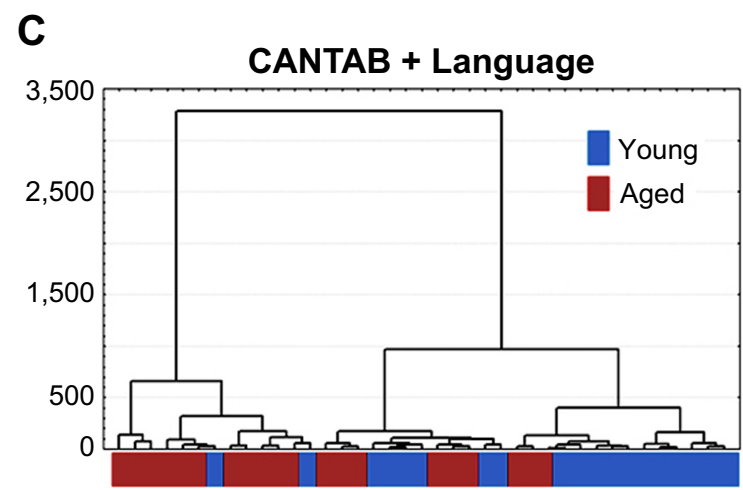

Figure I Dendrograms from cluster analysis applied to isolated or combined neuropsychological test results.

Notes: These analyses revealed three main clusters of distinct performances with variable degrees of contribution from young and older adults as a function of the neuropsychological test results submitted to the analysis. (A) Cluster analysis using CANTAB test results in isolation. Performances were mostly segregated into three distinct clusters: two dominated mostly by aged subjects, and one mostly by young subjects. (B) Language test results in isolation. (C) CANTAB + Language test results in combination. Note that cluster analysis using CANTAB test results in isolation revealed better distinction between young adult and older adult performances. Red and blue colors distinguish older and young adults respectively.

Abbreviation: CANTAB, Cambridge Neuropsychological Tests Automated Battery. 
Table I Probability density values (P-values) from discriminant analysis to detect which neuropsychological tests best contributed to cluster formation, either in isolation or in combination

\begin{tabular}{llll}
\hline Neuropsychological tests & CANTAB + Language & CANTAB & Language \\
\hline RVP latency & $4 \times 10^{-12}$ & $1 \times 10^{-10}$ & - \\
RTI SAS & $\mathrm{NS}$ & 0.030 & - \\
SWM strategy & 0.032 & $\mathrm{NS}$ & - \\
Concision & 0.010 & - & $\mathrm{NS}$ \\
Information units & 0.027 & - & $\mathrm{NS}$ \\
Metaphors & - & - & $4 \times 10^{-8}$ \\
Emotional prosody & - & - & 0.021 \\
\hline
\end{tabular}

Abbreviations: CANTAB, Cambridge Neuropsychological Tests Automated Battery; MMSE, Mini Mental State Examination; NS, nonsignificant; RTI, reaction time; RVP, Rapid Visual Information Processing; SAS, simple accuracy score; SWM, Spatial Working Memory.

Graphical representations in Figures 3 and 4 illustrate individual performances and mean values of the neuropsychological test results that were indicated by discriminant analysis to be the best predictors for cluster formation. Although the mean values of young and aged adults in these tests differed significantly, the dispersion of individual scores revealed remarkable similarities between some of the young and aged adults' performances, particularly in the following language tests: information units $(4.44 \pm 0.2$ vs $3.41 \pm 0.23$, mean \pm SEM), metaphors ( $40.89 \pm 2.03$ vs $35.58 \pm 1.67)$, and
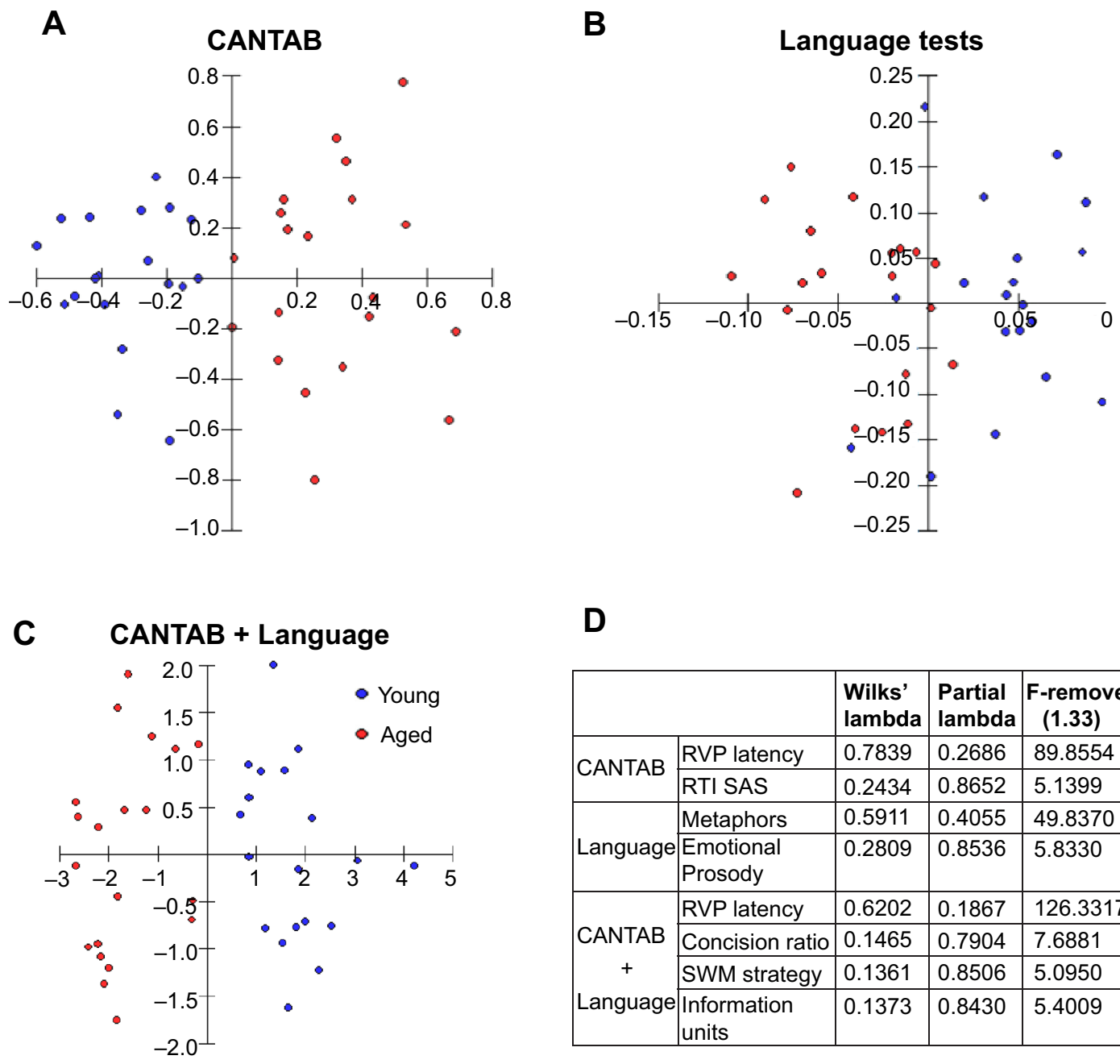

D

\begin{tabular}{|c|c|c|c|c|c|c|}
\hline & & $\begin{array}{l}\text { Wilks' } \\
\text { lambda }\end{array}$ & $\begin{array}{l}\text { Partial } \\
\text { lambda }\end{array}$ & $\begin{array}{c}\text { F-remove } \\
(1.33)\end{array}$ & P-level & Toler \\
\hline \multirow{2}{*}{ CANTAB } & RVP latency & 0.7839 & 0.2686 & 89.8554 & 0.0000 & 0.5598 \\
\hline & RTI SAS & 0.2434 & 0.8652 & 5.1399 & 0.0301 & 0.6856 \\
\hline \multirow[b]{2}{*}{ Language } & Metaphors & 0.5911 & 0.4055 & 49.8370 & \begin{tabular}{|l|}
0.0000 \\
\end{tabular} & 0.9423 \\
\hline & \begin{tabular}{|l|} 
Emotional \\
Prosody
\end{tabular} & 0.2809 & 0.8536 & 5.8330 & 0.0213 & 0.8347 \\
\hline \multirow{4}{*}{$\begin{array}{c}\text { CANTAB } \\
+ \\
\text { Language }\end{array}$} & RVP latency & 0.6202 & 0.1867 & 126.3317 & 0.0000 & 0.3895 \\
\hline & Concision ratio & 0.1465 & 0.7904 & 7.6881 & 0.0096 & 0.5120 \\
\hline & SWM strategy & 0.1361 & 0.8506 & 5.0950 & 0.0317 & 0.5751 \\
\hline & $\begin{array}{l}\text { Information } \\
\text { units }\end{array}$ & 0.1373 & 0.8430 & 5.4009 & 0.0273 & 0.3624 \\
\hline
\end{tabular}

Figure 2 Graphic representations of forward stepwise discriminant function analysis based on isolated (A, B) or combined CANTAB and Language (C) tests results. Notes: Note that the combined results discriminated better between young and older adult's performances. RVP latency, concision ratio, SWM strategy, and Information units were the variables that better contributed to cluster formation (D).

Abbreviations: CANTAB, Cambridge Neuropsychological Tests Automated Battery; RTI, reaction time; RVP, Rapid Visual Information Processing; SAS, simple accuracy score; SWM, Spatial Working Memory. 


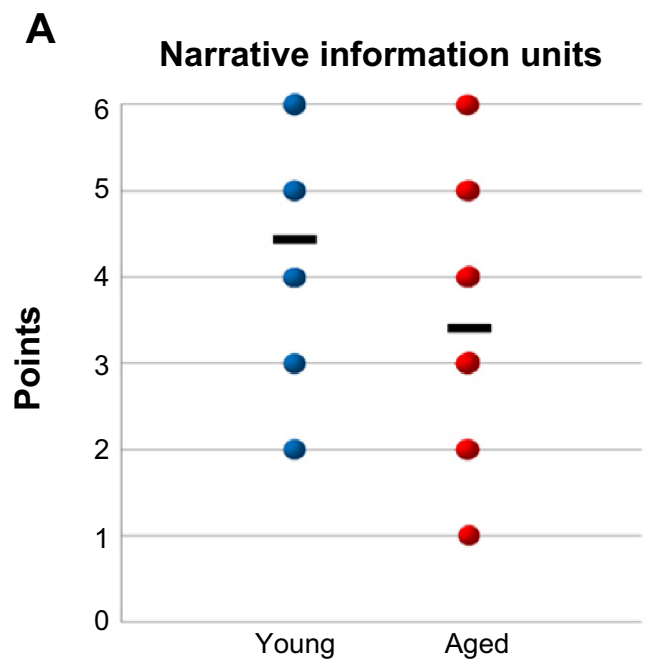

C

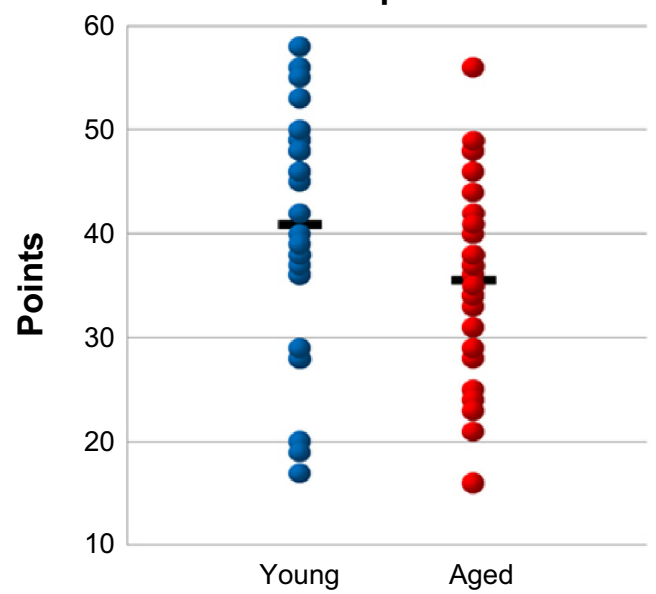

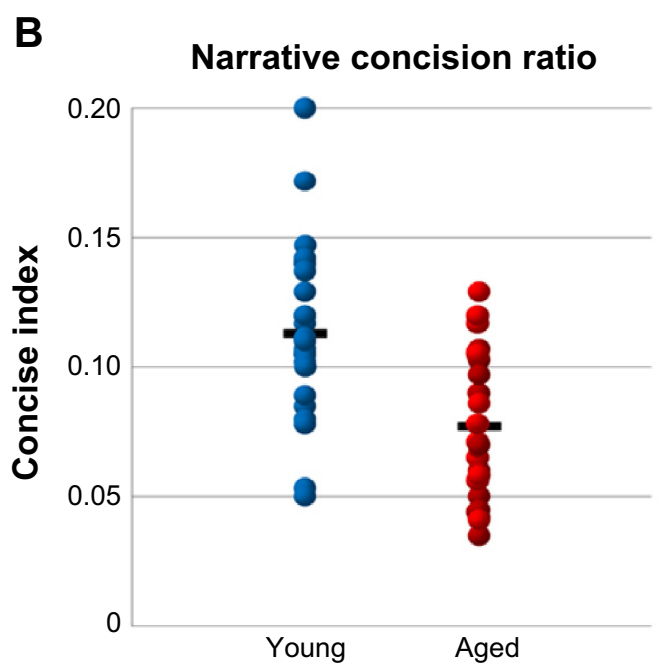

D Understanding emotional prosody

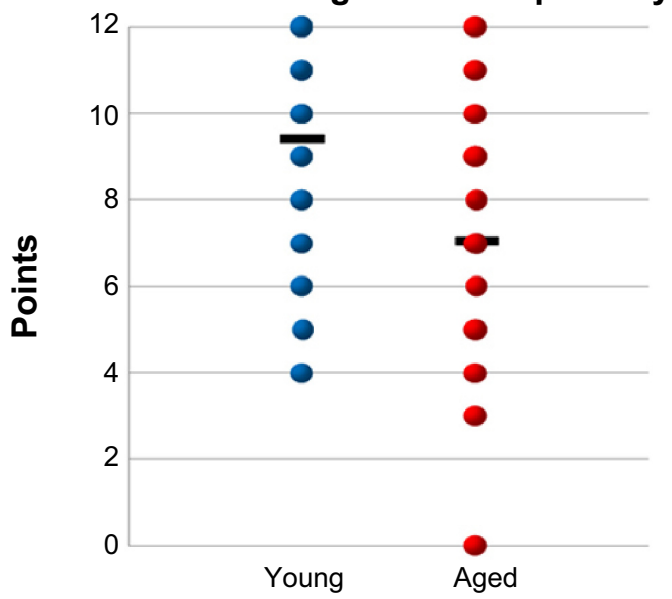

Figure 3 Graphic representations of mean scores and dispersion from the language test performances of young and older adults.

Notes: Narrative results based on information units (A), concision ratio (B), metaphors (C), and emotional prosody (D) revealed different degrees of dispersion, although the average values of older and young adults' performances differed significantly. Mean values are indicated by black dots in both young (blue circles) and older (red circles) groups. Note the smaller dispersion in the concision ratio results from the narrative tests.

emotional prosody $(9.41 \pm 0.4$ vs $7.06 \pm 0.45)$. These findings suggest that CANTAB tests are able to distinguish subtle differences in cognitive performances inside each group and between groups more accurately.

In addition, the probability of error given correctly from delayed matching to sample (DMS-PEGC) and total errors adjusted from paired associates learning (PAL-TEA) tests results (not illustrated) revealed significant differences (two tailed $t$-test $P<0.05$ ) between young and older adult groups $(\mathrm{PEGC}=0.13 \pm 0.03 \mathrm{vs}$ $0.26 \pm 0.02$ and TEA $=16 \pm 1.66$ vs $63.75 \pm 7.46$ ).

\section{Cluster and discriminant analysis for aged groups}

Similar analysis was applied to isolated neuropsychological test results for each age group. CANTAB, but not language tests, distinguished subgroups in the aged group, and the variables that best contributed to the clusters formation were RVP latency $(P=0.000009)$ and RVP A' (signal detection measure of sensitivity to the target $)(P=0.01)$ and Euclidian distance above 500 units. When applied in combination, CANTAB and language tests did not improve the distinction between subgroups.

\section{Cluster and discriminant analysis for young groups}

Language or CANTAB tests were not able to distinguish subgroups in the young group. Indeed, Euclidian distances revealed subgroups much closer to each other (Euclidian distances lower than 300 units).

A correlational matrix was employed to test for possible linear correlations between CANTAB and language assessments. No simple correlations were observed between the test results. 
A

\section{RVP latency}

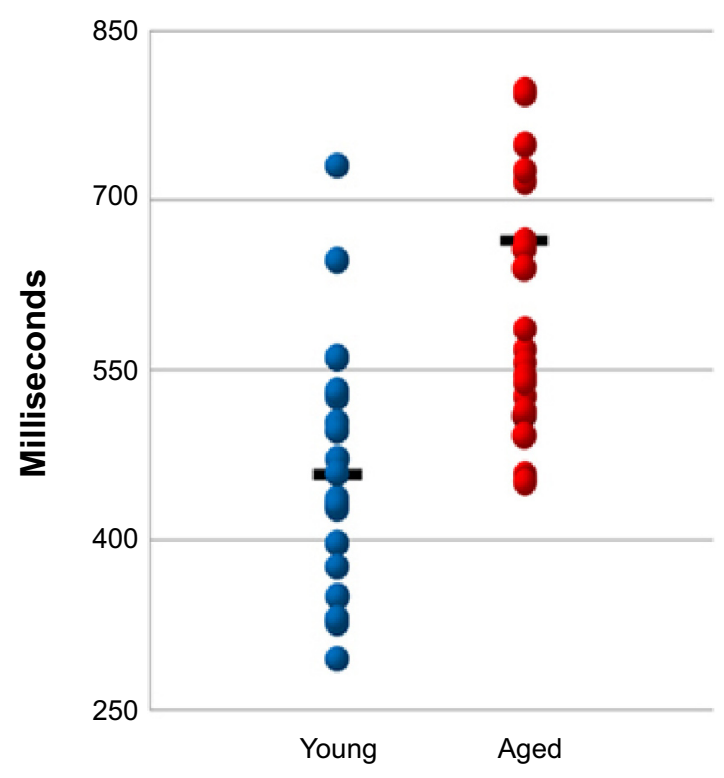

B

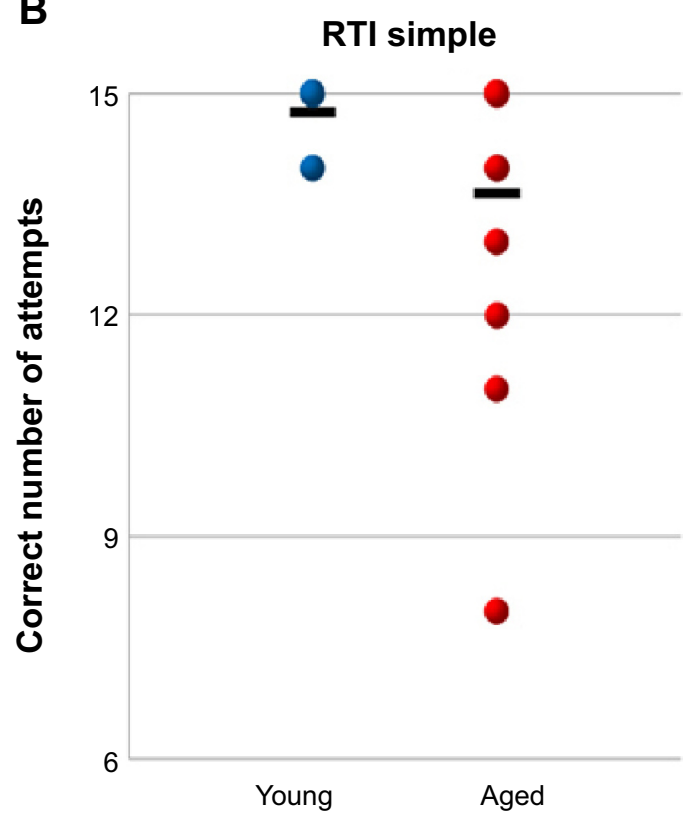

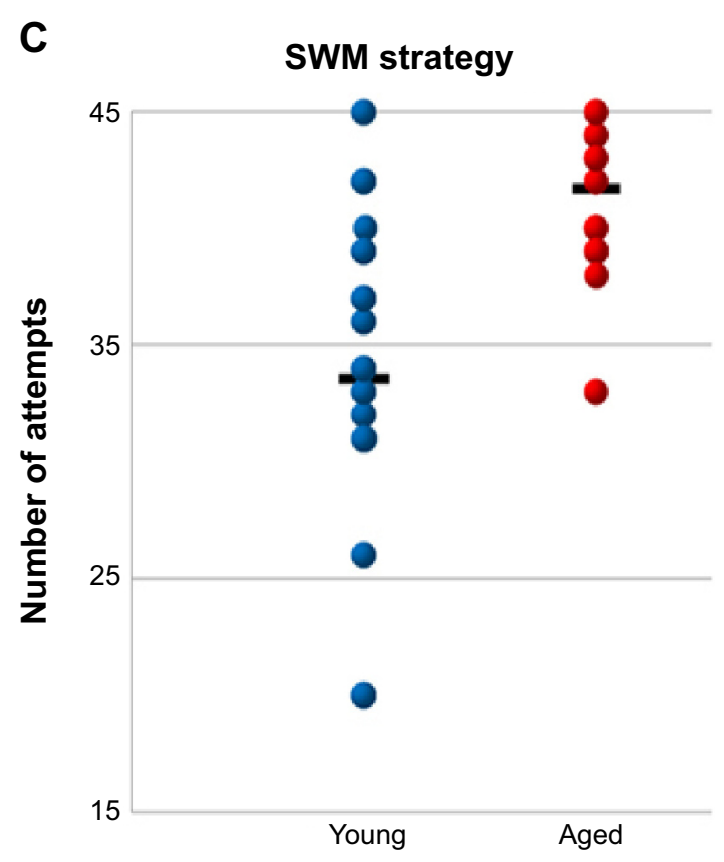

Figure 4 Graphic representations of mean scores and dispersion from CANTAB performances of young and older adults.

Notes: Results based on RVP latency (A), RTI simple (B), SWM strategy (C), revealed different degrees of dispersion, although the average values of older and young adults' performances differed significantly. Mean values are indicated by black dots in both young (blue circles) and older (red circles) groups. Note the smaller dispersion in the young adult's performances on RTI simple from CANTAB.

Abbreviations: CANTAB, Cambridge Neuropsychological Tests Automated Battery; RTI, reaction time; RVP, Rapid Visual Information Processing; SWM, Spatial Working Memory.

\section{Discussion}

In the present work, we investigated to what extent language and CANTAB tests, in isolation or in combination, could distinguish subtle differences in cognitive performances in young adults and elderly normal subjects and whether such performances on those tests correlated with each other.
Cluster and discriminant analysis revealed that CANTAB tests could distinguish between the cognitive performances of age groups better than language tests, and no linear correlations were observed between $\mathrm{CANTAB}$ and language results. We suggest that the use of large-scale automated visuospatial tests to assess learning and memory from CANTAB improves 
the signal-to-noise ratio, expanding our ability to define the limits between normal and pathological aging.

\section{CANTAB versus language tests to detect age-related cognitive decline}

The decline in cognitive function during human aging is well documented. This decline is apparent from tests of learning and memory, executive functions, attention, and processing speed. ${ }^{30,46}$ Neurophysiological and neuroanatomical evidence obtained through brain imaging suggests that the earliest brain changes occur in the prefrontal and temporal areas. The prefrontal area involves impairments in executive and planning functions, while the temporal areas involve memory and learning. ${ }^{47,48}$ In this study, cluster analysis revealed that selected CANTAB tests in isolation provided the best option to distinguish between the performances of the whole sample. In fact, the performances were mostly segregated into three distinct clusters: two dominated mostly by aged subjects and one mostly by young subjects (Figure 1A). Compared with cluster analysis based on isolated CANTAB results, all other dendrograms from both isolated (language tests) and combined tests (CANTAB + Language), revealed a lower degree of segregation.

In the specific case of aging, previous results from CANTAB tests have revealed significant cognitive decline as a function of age, limited to the SWM test (which assesses the frontal lobe) and the PAL test (which evaluates both frontal and the temporal lobe functions) ${ }^{49}$ Although the CANTAB DMS test also discriminated between the performances of young and older adults (not illustrated), it did not do as well as the PAL and SWM tests. However, because of its selectivity for temporal lobe function, lower performances of older adults on the DMS test suggest that episodic memory is indeed affected by age in our sample. In addition, the lower SWM performance in the group of older adults confirmed previous results using this test, demonstrating a decline in working memory with increasing age. ${ }^{30,50,51}$ In addition, and more importantly because of their discriminative properties in the cluster analysis, other CANTAB test results (RTI and RVP) were also affected by aging, suggesting that reduced processing speed (which appears to progress linearly with age) is associated with increased latency and reaction time. ${ }^{52}$

\section{Emotional and linguistic prosody}

Although impairments in linguistic and emotional prosody in healthy elderly individuals appear to be largely unrelated to cognitive decline, their integrity may be relevant to explore aging effects related to cognitive appraisal when comparing young versus old adults and measure right hemisphere cognitive decline in senescence.$^{53}$ In this work, as expected, we did not find any linear correlation between linguistic or emotional prosody scores and all other tests scores. These results are in agreement with others ${ }^{54-58}$ which demonstrate that the decline of linguistic and emotional prosody in the elderly is not related to age-related cognitive decline.

Interpretation of the affective state of others with respect to both speech and emotional face recognition is an essential component of normal psychosocial interactions. In agreement with previous reports, ${ }^{54,55,59,60}$ our findings demonstrate age-related decline in the perception of emotional prosody. As expected, the healthy elderly individuals performed significantly worse than young adults on tasks that assessed the comprehension of affective prosody.

The linguistic prosody test is used to measure the capacity to distinguish declarative statements and questions. Statements are characterized by falling terminal pitch, and questions by rising terminal pitch. ${ }^{60,61}$ Previous results demonstrated age-related difficulties in interpreting linguistic patterns,${ }^{56}$ and the performance of older adults on linguistic tasks with pitch contour patterns was less accurate than that of younger adults, ie, more difficulty identifying questions than statements. ${ }^{60}$ In agreement with these results, the present work confirms that, on average, young adults perform better than older adults with respect to linguistic prosody assessment. Similar to a previous report, ${ }^{60}$ older adults had slightly more impaired emotional prosody than linguistic prosody compared with young adults.

\section{Metaphors}

Literal lexical-semantic comprehension is always necessary in social communication, but it is not sufficient to guarantee appropriate communication. ${ }^{62}$ Comprehension of nonliteral implication is often essential to interpret the implicit meaning in a sentence, which is not always expressed explicitly. ${ }^{63}$ Although no consensus has been obtained regarding the decline in metaphor comprehension with aging, ${ }^{64-69}$ previous reports have demonstrated that young adults make fewer mistakes in metaphor tests than healthy older adults. ${ }^{70}$ In agreement with these findings, absolute numbers in the present report indicated that older adults committed an average of $13 \%$ more errors on the metaphor test than younger adults. As described for emotional prosody, we detected no correlations between performance on the metaphor test and age-related cognitive decline in the present work. 
Another group of language functions was also impaired by age. This finding is indicated by the lower scores of older adults versus younger adults on the Boston Naming Test (reduced version), linguistic prosody, and narrative tests (main concepts, information units, and concision).

\section{Boston Naming Test}

The Boston Naming Test is able to assess the degree of difficulty in finding words encountered by healthy elderly individuals, as well as by individuals in the initial stages of dementia. ${ }^{71}$ Although the results of the confrontational Boston Naming Test (as applied in the present work) are qualitatively affected by the cultural background, particularly with regard to non-English-speaking populations, their quantitative scores are not. ${ }^{72}$ Thus, as previously described for non-native English speakers, ${ }^{72}$ our findings using the Boston Naming Test version adapted to speakers of Brazilian Portuguese $^{73}$ demonstrated significantly lower scores among older adults than among young adults.

\section{Narrative tests}

In general discourse, comprehension abilities decline with age $^{74,75}$ and appear to be influenced by executive functions. ${ }^{76}$ Age-related changes have also been reported in the production of discourse, and a picture description task is frequently used to reveal such decline. Nonpathological aging is typically associated with dysfunctions of the prefrontal cortex. ${ }^{77,78}$ Because discourse production is thought to depend on prefrontal cortical integrity, it has been proposed that the analysis of narrative discourse structure may be a measure of executive function in adults. ${ }^{76}$ Although we have not yet established cutoff points for the Brazilian population using narrative tests and large samples, we found (as previously described in Ref. 79) that, on average, the narrative structure was significantly impaired in older adults compared with young adults. Older adults exhibited lower scores in main concepts, information units, and concision than young adults.

\section{CANTAB versus language tests: limits of comparative analysis}

To be reliable, neuropsychological tests must replicate results in a systematic way when subjects are tested repeatedly. To be valid, the tests must be able to identify individuals with the same type of neuroanatomical change, who exhibit similar and consistent test performances for the same cognitive domain and with the same sensitivity. To be specific, the tests must be sensitive to the functional changes of interest, but not to others. ${ }^{49}$ Because these conditions were met and consistent results were obtained for a number of selected tests intended to assess the prefrontal and temporal lobes, ${ }^{50,51}$ the CANTAB was considered reliable in assessing the type and degree of functional loss and the specificity of aging-associated changes in the temporal and prefrontal lobes. ${ }^{80,81}$ Because a large number of individuals were assessed by the CANTAB, it was possible to establish the degree of correlation between the functional demand imposed by the selected tests and the neural areas involved in those functions. ${ }^{49-51}$

Although we do not yet have a large sample to define the norms and cutoff points for the Brazilian population using the CANTAB, this battery (originally designed to compare monkey and human performances ${ }^{48,82-84}$ ) is largely independent of cultural differences. ${ }^{19,30,84,85}$ Indeed, our findings are in agreement with a number of previous studies with CANTAB ${ }^{27,30,50,51}$ which confirm age-related cognitive decline in selected tests for both temporal and prefrontal lobe functions. Because the neuropsychological test results from the CANTAB have been validated in many countries with different cultures, ${ }^{30,51,86,87}$ we suggest that the CANTAB may be an adequate tool for transnational comparative studies of both normal and pathological aging.

However, it is important to mention that the selected CANTAB tests did not include verbal measures. Thus, comparative analysis with the language tests detailed in this paper may have some limitations in the sense that similar cognitive domains in the CANTAB and language tests are not necessarily processed and retrieved using the same subjacent neural networks and mechanisms. ${ }^{88}$ In addition, possible correlations between CANTAB subtests and traditional paper-and-pencil neuropsychological tests demonstrated modest associations when education and age were controlled for. ${ }^{27}$ In agreement with these observations, our findings revealed no linear correlation between the results of language and CANTAB tests in the present work.

Finally, it has been suggested that familiarity with the computer interface may influence performance on computerized test batteries; ${ }^{89}$ however, because the CANTAB does not require any computer/technology knowledge, frequent computer users do not gain any significant operative advantage over users who are unfamiliar with computerized automated tests. ${ }^{90}$

Although the number of individuals tested in the present study does not encourage generalized conclusions, our exploratory study strongly suggests that the CANTAB test results in isolation may improve the signal-to-noise ratio when distinguishing the performance of subgroups both in young adults and the elderly better than language 
tests do. Because the language and CANTAB tests assessing corresponding cognitive domains were not linearly correlated, it is important that we increase our sample size in order to be able to confirm and generalize these preliminary observations.

\section{Acknowledgments}

This work was supported by the Programa Pesquisa para o SUS: gestão compartilhada em saúde (PPSUS) - Ministério da Saúde/Conselho Nacional de Desenvolvimento Científico e Tecnológico (CNPq)/Fundação de Amparo à Pesquisa do Estado do Pará (FAPESPA)/Secretaria de Saúde do Estado do Pará (SESPA) (Grant No 051/2007 and 013/2009); Agência Brasileira da Inovação (FINEP)/Fundação de Amparo e Desenvolvimento da Pesquisa (FADESP) (Grant No 01.04.0043.00); and Pró-Reitoria de Pesquisa (PROPESP-UFPA)/Fundação de Amparo e Desenvolvimento da Pesquisa (FADESP).

\section{Disclosure}

The authors report no conflicts of interest in this work.

\section{References}

1. Fratiglioni L, De Ronchi D, Agüero-Torres H. Worldwide prevalence and incidence of dementia. Drugs Aging. 1999;15:365-375.

2. Zhu CW, Sano M. Economic considerations in the management of Alzheimer's disease. Clin Interv Aging. 2006;1:143-154.

3. Ernst RL, Hay JW, Fenn C, Tinklenberg J, Yesavage JA. Cognitive function and the costs of Alzheimer disease. An exploratory study. Arch Neurol. 1997;54:687-693.

4. Daffner KR. Promoting successful cognitive aging: a comprehensive review. J Alzheimers Dis. 2010;19:1101-1122.

5. Wagster MV. Cognitive aging research: an exciting time for a maturing field: a postscript to the special issue of neuropsychology review. Neuropsychol Rev. 2009;19:523-525.

6. Folstein MF, Folstein SE, McHugh PR. "Mini-mental state". A practical method for grading the cognitive state of patients for the clinician. J Psychiatr Res. 1975;12:189-198.

7. Franco-Marina F, García-González JJ, Wagner-Echeagaray F, et al. The mini-mental state examination revisited: ceiling and floor effects after score adjustment for educational level in an aging Mexican population. Int Psychogeriatr. 2010;22:72-81.

8. Marioni RE, Chatfield M, Brayne C, Matthews FE; Medical Research Council Cognitive Function and Ageing Study Group. The reliability of assigning individuals to cognitive states using the Mini Mental-State Examination: a population-based prospective cohort study. BMC Med Res Methodol. 2011;11:127.

9. Brayne C. The mini-mental state examination, will we be using it in 2001? Int J Geriatr Psychiatry. 1998;13:285-290.

10. Tombaugh GC, Yang SH, Swanson RA, Sapolsky RM. Glucocorticoids exacerbate hypoxic and hypoglycemic hippocampal injury in vitro: biochemical correlates and a role for astrocytes. J Neurochem. 1992;59: 137-146.

11. Blacker D, Lee H, Muzikansky A, et al. Neuropsychological measures in normal individuals that predict subsequent cognitive decline. Arch Neurol. 2007;64:862-871.

12. Howieson DB, Dame A, Camicioli R, Sexton G, Payami H, Kaye JA. Cognitive markers preceding Alzheimer's dementia in the healthy oldest old. J Am Geriatr Soc. 1997;45:584-589.
13. Small GW, La Rue A, Komo S, Kaplan A, Mandelkern MA. Predictors of cognitive change in middle-aged and older adults with memory loss. Am J Psychiatry. 1995;152:1757-1764.

14. Tierney MC, Szalai JP, Snow WG, et al. Prediction of probable Alzheimer's disease in memory-impaired patients: a prospective longitudinal study. Neurology. 1996;46:661-665.

15. Rubin EH, Storandt M, Miller JP, et al. A prospective study of cognitive function and onset of dementia in cognitively healthy elders. Arch Neurol. 1998;55:395-401.

16. Chen P, Ratcliff G, Belle SH, Cauley JA, DeKosky ST, Ganguli M. Cognitive tests that best discriminate between presymptomatic AD and those who remain nondemented. Neurology. 2000;55:1847-1853.

17. Blackwell AD, Sahakian BJ, Vesey R, Semple JM, Robbins TW, Hodges JR. Detecting dementia: novel neuropsychological markers of preclinical Alzheimer's disease. Dement Geriatr Cogn Disord. 2004;17: $42-48$.

18. Belleville S, Gauthier S, Lepage E, Kergoat MJ, Gilbert B. Predicting decline in mild cognitive impairment: a prospective cognitive study. Neuropsychology. 2014;28(4):643-652.

19. Weissberger GH, Salmon DP, Bondi MW, Gollan TH. Which neuropsychological tests predict progression to Alzheimer's disease in Hispanics? Neuropsychology. 2013;27:343-355.

20. Clark DG, Wadley VG, Kapur P, et al. Lexical factors and cerebral regions influencing verbal fluency performance in MCI. Neuropsychologia. 2014;54:98-111.

21. Pakhomov SV, Hemmy LS, Lim KO. Automated semantic indices related to cognitive function and rate of cognitive decline. Neuropsychologia. 2012;50:2165-2175.

22. Hayden KM, Kuchibhatla M, Romero HR, et al. Pre-clinical cognitive phenotypes for Alzheimer disease: a latent profile approach. Am J Geriatr Psychiatry. 2014;22(11):1364-1374.

23. Fowler KS, Saling MM, Conway EL, Semple JM, Louis WJ. Paired associate performance in the early detection of DAT. J Int Neuropsychol Soc. 2002;8:58-71.

24. Iachini I, Iavarone A, Senese VP, Ruotolo F, Ruggiero G. Visuospatial memory in healthy elderly, AD and MCI: a review. Curr Aging Sci. 2009;2:43-59.

25. Juncos-Rabadán O, Pereiro AX, Facal D, Reboredo A, Lojo-Seoane C. Do the Cambridge Neuropsychological Test Automated Battery episodic memory measures discriminate amnestic mild cognitive impairment? Int J Geriatr Psychiatry. 2014;29(6):602-609.

26. Wild K, Howieson D, Webbe F, Seelye A, Kaye J. Status of computerized cognitive testing in aging: a systematic review. Alzheimers Dement. 2008;4:428-437.

27. Smith PJ, Need AC, Cirulli ET, Chiba-Falek O, Attix DK. A comparison of the Cambridge Automated Neuropsychological Test Battery (CANTAB) with "traditional" neuropsychological testing instruments. J Clin Exp Neuropsychol. 2013;35:319-328.

28. Skolimowska J, Wesierska M, Lewandowska M, Szymaszek A, Szelag E. Divergent effects of age on performance in spatial associative learning and real idiothetic memory in humans. Behav Brain Res. 2011;218:87-93.

29. Junkkila J, Oja S, Laine M, Karrasch M. Applicability of the CANTAB-PAL computerized memory test in identifying amnestic mild cognitive impairment and Alzheimer's disease. Dement Geriatr Cogn Disord. 2012;34:83-89.

30. De Luca CR, Wood SJ, Anderson V, et al. Normative data from the CANTAB. I: development of executive function over the lifespan. J Clin Exp Neuropsychol. 2003;25:242-254.

31. Lowe C, Rabbitt P. Test/re-test reliability of the CANTAB and ISPOCD neuropsychological batteries: theoretical and practical issues. Cambridge Neuropsychological Test Automated Battery. International Study of Post-Operative Cognitive Dysfunction. Neuropsychologia. 1998;36: 915-923.

32. Sahakian BJ, Owen AM. Computerized assessment in neuropsychiatry using CANTAB: discussion paper. J R Soc Med. 1992;85:399-402.

33. Bertolucci PH, Brucki SM, Campacci SR, Juliano Y. [The Mini-Mental State Examination in a general population: impact of educational status]. Arq Neuropsiquiatr. 1994;52:1-7. Portuguese. 
34. Marcopulos B, McLain C. Are our norms "normal"? A 4-year follow-up study of a biracial sample of rural elders with low education. Clin Neuropsychol. 2003;17:19-33.

35. Bertolucci PHF, Okamoto IH, Toniolo JN, Ramos LR, Bruki SMD. Desempenho da população brasileira na bateria neuropsicológica do Consortium to Establish a Registry for Alzheimer's Disease (CERAD). Rev Psiquiatr Clín. 1998;25:80-83. Portuguese.

36. Bertolucci PH, Okamoto IH, Brucki SM, Siviero MO, Toniolo Neto J, Ramos LR. Applicability of the CERAD neuropsychological battery to Brazilian elderly. Arq Neuropsiquiatr. 2001;59:532-536.

37. Caramelli P, Carthery-Goulart MT, Porto CS, Charchat-Fichman H, Nitrini R. Category fluency as a screening test for Alzheimer disease in illiterate and literate patients. Alzheimer Dis Assoc Disord. 2007;21: 65-67.

38. Forbes-McKay KE, Venneri A. Detecting subtle spontaneous language decline in early Alzheimer's disease with a picture description task. Neurol Sci. 2005;26:243-254.

39. Groves-Wright K, Neils-Strunjas J, Burnett R, O’Neill MJ. A comparison of verbal and written language in Alzheimer's disease. J Commun Disord. 2004;37:109-130.

40. Fonseca RP, Joanette Y, Cote H, et al. Brazilian version of the Protocole Montreal d'Evaluation de la Communication (Protocole MEC) normative and reliability data. Span J Psychol. 2008;11:678-688.

41. Fonseca RP, Parente MA, Côté H, Ska B, Joanette Y. Introducing a communication assessment tool to Brazilian speech therapists: the MAC Battery. Pro Fono. 2008;20:285-291.

42. Steele GE, Weller RE. Qualitative and quantitative features of axons projecting from caudal to rostral inferior temporal cortex of squirrel monkeys. Vis Neurosci. 1995;12:701-722.

43. Schweitzer L, Renehan WE. The use of cluster analysis for cell typing. Brain Res Brain Res Protoc. 1997;1:100-108.

44. Gomes-Leal W, Silva GJ, Oliveira RB, Picanco-Diniz CW. Computerassisted morphometric analysis of intrinsic axon terminals in the supragranular layers of cat striate cortex. Anat Embryol (Berl). 2002;205 291-300.

45. Rocha EG, Santiago LF, Freire MAM, et al. Callosal axon arbors in the limb representations of the somatosensory cortex (SI) in the agouti (Dasyprocta primnolopha). J Comp Neurol. 2007;500:255-266.

46. Sliwinski M, Buschke H. Cross-sectional and longitudinal relationships among age, cognition, and processing speed. Psychol Aging. 1999;14: 18-33.

47. Nagahara AH, Nicolle MM, Gallagher M. Alterations in [3H]-kainate receptor binding in the hippocampal formation of aged Long-Evans rats. Hippocampus. 1993;3:269-277.

48. Nagahara AH, Bernot T, Tuszynski MH. Age-related cognitive deficits in rhesus monkeys mirror human deficits on an automated test battery. Neurobiol Aging. 2010;31:1020-1031.

49. Rabbitt P, Lowe C. Patterns of cognitive ageing. Psychol Res. 2000;63: 308-316.

50. Robbins TW, James M, Owen AM, et al. A study of performance on tests from the CANTAB battery sensitive to frontal lobe dysfunction in a large sample of normal volunteers: implications for theories of executive functioning and cognitive aging. $J$ Int Neuropsychol Soc. 1998;4:474-490.

51. Robbins TW, James M, Owen AM, Sahakian BJ, McInnes L, Rabbitt P Cambridge Neuropsychological Test Automated Battery (CANTAB): a factor analytic study of a large sample of normal elderly volunteers. Dementia. 1994;5:266-281.

52. Carriere JS, Cheyne JA, Solman GJ, Smilek D. Age trends for failures of sustained attention. Psychol Aging. 2010;25:569-574.

53. Ross ED, Monnot M. Affective prosody: what do comprehension errors tell us about hemispheric lateralization of emotions, sex and aging effects, and the role of cognitive appraisal. Neuropsychologia. 2011;49(5):866-877.

54. Orbelo DM, Grim MA, Talbott RE, Ross ED. Impaired comprehension of affective prosody in elderly subjects is not predicted by age-related hearing loss or age-related cognitive decline. J Geriatr Psychiatry Neurol. 2005;18:25-32.
55. Kiss I, Ennis T. Age-related decline in perception of prosodic affect. Appl Neuropsychol. 2001;8:251-254.

56. Allen R, Brosgole L. Facial and auditory affect recognition in senile geriatrics, the normal elderly and young adults. Int J Neurosci. 1993;68: 33-42.

57. Cohen ES, Brosgole L. Visual and auditory affect recognition in senile and normal elderly persons. Int J Neurosci. 1988;43:89-101.

58. Tompkins CA, Flowers CR. Perception of emotional intonation by brain-damaged adults: the influence of task processing levels. $J$ Speech Hear Res. 1985;28:527-538.

59. Ruffman T, Henry JD, Livingstone V, Phillips LH. A meta-analytic review of emotion recognition and aging: implications for neuropsychological models of aging. Neurosci Biobehav Rev. 2008;32:863-881.

60. Mitchell RL, Kingston RA, Barbosa Bouças SL. The specificity of age-related decline in interpretation of emotion cues from prosody. Psychol Aging. 2011;26:406-414.

61. Srinivasan RJ, Massaro DW. Perceiving prosody from the face and voice: distinguishing statements from echoic questions in English. Lang Speech. 2003;46:1-22.

62. Russell RL. Social communication impairments: pragmatics. Pediatr Clin North Am. 2007;54:483-506; vi.

63. Amodio DM, Frith CD. Meeting of minds: the medial frontal cortex and social cognition. Nat Rev Neurosci. 2006;7:268-277.

64. Maki Y, Yamaguchi T, Koeda T, Yamaguchi H. Communicative competence in Alzheimer's disease: metaphor and sarcasm comprehension. Am J Alzheimers Dis Other Demen. 2013;28:69-74.

65. Amanzio M, Geminiani G, Leotta D, Cappa S. Metaphor comprehension in Alzheimer's disease: novelty matters. Brain Lang. 2008; 107:1-10

66. Mashal N, Gavrieli R, Kavé G. Age-related changes in the appreciation of novel metaphoric semantic relations. Neuropsychol Dev Cogn B Aging Neuropsychol Cogn. 2011;18:527-543.

67. Szuchman LT, Erber JT. Young and older adults' metaphor interpretation: the judgments of professionals and nonprofessionals. Exp Aging Res. 1990;16:67-72.

68. Newsome MR, Glucksberg S. Older adults filter irrelevant information during metaphor comprehension. Exp Aging Res. 2002;28:253-267.

69. Gregory ME, Waggoner JE. Factors that influence metaphor comprehension skills in adulthood. Exp Aging Res. 1996;22:83-98.

70. Morrone I, Declercq C, Novella JL, Besche C. Aging and inhibition processes: the case of metaphor treatment. Psychol Aging. 2010;25: 697-701.

71. Tombaugh TN, Hubley AM. The 60-item Boston Naming Test: norms for cognitively intact adults aged 25 to 88 years. J Clin Exp Neuropsychol. 1997;19:922-932.

72. Chen TB, Lin CY, Lin KN, et al. Culture qualitatively but not quantitatively influences performance in the Boston naming test in a Chinese-speaking population. Dement Geriatr Cogn Dis Extra. 2014;4: 86-94.

73. Miotto EC, Sato J, Lucia MC, Camargo CH, Scaff M. Development of an adapted version of the Boston Naming Test for Portuguese speakers. Rev Bras Psiquiatr. 2010;32:279-282.

74. McGinnis D. Text comprehension products and processes in young, young-old, and old-old adults. J Gerontol B Psychol Sci Soc Sci. 2009; 64:202-211.

75. Wright HH, Capilouto GJ, Srinivasan C, Fergadiotis G. Story processing ability in cognitively healthy younger and older adults. J Speech Lang Hear Res. 2011;54:900-917.

76. Cannizzaro MS, Coelho CA. Analysis of narrative discourse structure as an ecologically relevant measure of executive function in adults. J Psycholinguist Res. 2013;42:527-549.

77. Motes MA, Biswal BB, Rypma B. Age-dependent relationships between prefrontal cortex activation and processing efficiency. Cogn Neurosci. 2011;2:1-10.

78. Tisserand DJ, Jolles J. On the involvement of prefrontal networks in cognitive ageing. Cortex. 2003;39:1107-1128.

79. Ardila A, Rosselli M. Spontaneous language production and aging: sex and educational effects. Int J Neurosci. 1996;87:71-78. 
80. Chase HW, Clark L, Sahakian BJ, Bullmore ET, Robbins TW. Dissociable roles of prefrontal subregions in self-ordered working memory performance. Neuropsychologia. 2008;46:2650-2661.

81. de Rover M, Pironti VA, McCabe JA, et al. Hippocampal dysfunction in patients with mild cognitive impairment: a functional neuroimaging study of a visuospatial paired associates learning task. Neuropsychologia. 2011;49:2060-2070.

82. Spinelli S, Pennanen L, Dettling AC, Feldon J, Higgins GA, Pryce CR. Performance of the marmoset monkey on computerized tasks of attention and working memory. Brain Res Cogn Brain Res. 2004;19: 123-137.

83. Rodriguez JS, Zurcher NR, Bartlett TQ, Nathanielsz PW, Nijland MJ. CANTAB delayed matching to sample task performance in juvenile baboons. J Neurosci Methods. 2011;196:258-263.

84. Zürcher NR, Rodriguez JS, Jenkins SL, et al. Performance of juvenile baboons on neuropsychological tests assessing associative learning, motivation and attention. J Neurosci Methods. 2010;188:219-225.
85. Simpson EE, Maylor EA, Rae G, et al. Cognitive function in healthy older European adults: the ZENITH study. Eur J Clin Nutr. 2005; 59(suppl 2):S26-S30.

86. Lee A, Archer J, Wong CK, Chen SH, Qiu A. Age-related decline in associative learning in healthy Chinese adults. PLoS One. 2013;8: e80648.

87. McPhee JS, Hogrel JY, Maier AB, et al. Physiological and functional evaluation of healthy young and older men and women: design of the European MyoAge study. Biogerontology. 2013;14:325-337.

88. 88. Salthouse TA. Aging and measures of processing speed. Biol Psychol. 2000;54:35-54.

89. Iverson GL, Brooks BL, Ashton VL, Johnson LG, Gualtieri CT. Does familiarity with computers affect computerized neuropsychological test performance? J Clin Exp Neuropsychol. 2009;31:594-604.

90. Steves CJ, Jackson SH, Spector TD. Cognitive change in older women using a computerised battery: a longitudinal quantitative genetic twin study. Behav Genet. 2013;43:468-479.
Clinical Interventions in Aging

\section{Publish your work in this journal}

Clinical Interventions in Aging is an international, peer-reviewed journal focusing on evidence-based reports on the value or lack thereof of treatments intended to prevent or delay the onset of maladaptive correlates of aging in human beings. This journal is indexed on PubMed Central, MedLine,

\section{Dovepress}

CAS, Scopus and the Elsevier Bibliographic databases. The manuscript management system is completely online and includes a very quick and fair peer-review system, which is all easy to use. Visit http://www.dovepress. com/testimonials.php to read real quotes from published authors. 